
\title{
28 Research Square \\ EYA4 could be an indicator signifying colorectal cancer sufferers' prognoses
}

\section{Yang Yan}

Chinese PLA General Hospital

Xiaohui Du ( $\sim$ bdihefs@yeah.net)

Chinese PLA General Hospital https://orcid.org/0000-0001-7127-3610

\section{Shaoyou Xia}

Chinese PLA General Hospital

\section{Songyan Li}

Chinese PLA General Hospital

\section{Da Teng}

Chinese PLA General Hospital

\section{Shidong $\mathrm{Hu}$}

Chinese PLA General Hospital

\section{Yufeng Wang}

Chinese PLA General Hospital

\section{Rong Li}

Chinese PLA General Hospital

\section{Research article}

Keywords: Eyes absent 4 (EYA4), Colorectal cancer, Prognosis

Posted Date: July 24th, 2020

DOI: https://doi.org/10.21203/rs.3.rs-46920/v1

License: (c) (i) This work is licensed under a Creative Commons Attribution 4.0 International License. Read Full License 


\section{Abstract \\ Background}

Eyes absent 4 (EYA4) is involved in various biological processes. The aim of this study was to investigate the expression of EYA4 and its prognostic value in colorectal cancer (CRC).

\section{Methods}

The mRNA level of EYA4 in diseased tissues and adjacent normal tissues of CRC patients were detected by quantitative real-time polymerase chain reaction (qRT-PCR). The association between EYA4 expression and clinicopathological characteristics was analyzed by $\chi^{2}$ test. Kaplan-Meier analysis with log rank test was performed to evaluate the effects of EYA4 expression on overall survival of CRC patients. Cox regression model was applied for prognosis analysis in CRC.

\section{Results}

The mRNA level of EYA4 was significantly decreased in CRC tissues compared with that in the adjacent normal tissue $(P<0.01)$. And its expression was affected by DUKE stage $(P=0.034)$, differentiation $(P=$ $0.027)$ and vascular invasion $(P=0.037)$. Survival curve showed that patients with low expression of EYA4 had a significantly shorter overall survival than those with high expression (log rank test, $P=0.008$ ). Low expression of EYA4 $(\mathrm{HR}=1.989,95 \% \mathrm{Cl}=1.090-3.62902, P=0.025)$ was an independent biomarker for poor prognosis in CRC patients.

\section{Conclusion}

EYA4 expression is decreased in CRC patients and negatively correlated with aggressive tumor progression. EYA4 may be a potential prognostic biomarker for CRC.

\section{Background}

Colorectal cancer (CRC) is one of the most common malignancies in the world, being responsible for many cancer-related deaths in adult [1]. The inflammatory bowel disease (IBD), especially chronic ulcerative colitis (UC) and Crohn's colitis, can enhance the risk of CRC [2,3]. Despite of the great improvements in surgical resections as well as the integration of chemoradiotherapy and targeted therapy, the long term survival of patients with CRC is still very poor [4,5]. Many CRC cases will develop local recurrence or distant metastasis within 5 years after operation, resulting in therapeutic failures and high mortality [6]. Until now, there is no widely accepted parameter to provide reliable information for tumor progression and prognosis in CRC [7]. For this reason, it is urgently needed to identify efficient cancer-related genetic biomarkers for improving the poor prognosis of CRC. 
The Eyes Absent 4 gene (EYA4), is a member of EYA gene family which includes four members, EYA1EYA4, in mammals [8]. The EYA family was initially identified in Drosophila, and played pivotal roles in eye development [9]. Growing evidences have reported that EYA4 is implicated in the formation and development of mammalian organs, regulation of cellular behaviors, apoptosis, innate immunity, DNA damage repair, angiogenesis, as well as tumorigenesis [10]. Recently the aberrant hypermethylation of EYA4 promoter has been observed in a number of cancers, such as melanomas, lung cancer, pancreatic cancer, and hepatocellular carcinoma [11-14]. It was reported that hypermethylation mediated EYA4 silence might contribute to tumorigenesis, revealing the inhibitory roles of EYA4 in carcinogenesis [14]. The anti-tumor action of EYA4 was also studied in CRC. Sung-Jin Kim et al, demonstrated that overexpression of EYA4 could lead to inhibition of cell proliferation via inducting up-regulation of $D K K 1$ and inhibiting the Wnt signaling pathway, indicating its capacity as a tumor suppressor gene in CRC [15]. However, the clinical significance of EYA4 in prognosis of CRC was rarely reported.

In this study, we mainly investigated the expression pattern of EYA4 in CRC tissues samples, and its association with clinicopathological characteristics of the patients. Additionally, we further estimated the prognostic value of EYA4 in CRC.

\section{Materials And Methods}

\section{Patients and specimens}

The study population was consisted of 155 patients pathologically diagnosed with CRC, including 71 females and 84 males. The tumor tissues and adjacent normal tissues specimens were collected from the patients through surgical resection at Chinese PLA General Hospital. None of these patients had received chemotherapy or radiation therapy prior to surgery. The specimens were immediately put into liquid nitrogen after surgery and then stored at $-80^{\circ} \mathrm{C}$ until RNA extraction. The detail clinical information of the patients was listed in Table 1. A 5-years follow-up was conducted for the patients. In our study, we excluded the patients who were died from unexpected events or other diseases. 
Table 1

The relationship between EYA4 expression and clinicopathological features in CRC patients

\begin{tabular}{|c|c|c|c|c|c|}
\hline \multirow[t]{2}{*}{ Characteristics } & \multirow{2}{*}{$\begin{array}{l}\text { Cases } \\
(n=155)\end{array}$} & \multicolumn{2}{|c|}{ EYA4 expression } & \multirow{2}{*}{$x^{2}$} & \multirow[t]{2}{*}{$P$} \\
\hline & & Low $(n=56)$ & High $(n=99)$ & & \\
\hline Age (years) & & & & 1.278 & 0.258 \\
\hline$<60$ & 73 & 23 & 50 & & \\
\hline$\geq 60$ & 82 & 33 & 49 & & \\
\hline Gender & & & & 0.792 & 0.374 \\
\hline Male & 84 & 33 & 51 & & \\
\hline Female & 71 & 23 & 48 & & \\
\hline Lymph node metastasis & & & & 3.653 & 0.056 \\
\hline Negative & 93 & 28 & 65 & & \\
\hline Positive & 62 & 28 & 34 & & \\
\hline DUKES clinical stage & & & & 4.511 & 0.034 \\
\hline$A+B$ & 92 & 27 & 65 & & \\
\hline$C+D$ & 63 & 29 & 34 & & \\
\hline Differentiation & & & & 4.875 & 0.027 \\
\hline median + well & 90 & 26 & 64 & & \\
\hline poor & 65 & 30 & 35 & & \\
\hline Tumor location & & & & 0.581 & 0.446 \\
\hline Colon & 92 & 31 & 61 & & \\
\hline Rectum & 63 & 25 & 38 & & \\
\hline Vascular invasion & & & & 4.332 & 0.037 \\
\hline Negative & 89 & 26 & 63 & & \\
\hline Positive & 66 & 30 & 36 & & \\
\hline
\end{tabular}

This study was approved by the Research Ethics Committee of Chinese PLA General Hospital. All the patients gave written informed consent prior to surgery. 


\section{Rna Extraction And Quantitative Real-time Polymerase Chain Reaction (qrt-pcr)}

Total RNA was isolated from tissues specimens using Trizol reagent (Invitrogen, Carlsbad, CA) and reversely transcribed into cDNA by the RevertAid First Strand cDNA Synthesis Kits (Fermentas, Lithuania) according to the manufacturer's protocol. The relative expression value of EYA4 was evaluated by qRTPCR reaction and CDNA samples served as amplification template. The reaction was carried out using SYBR Premix Ex Taq ${ }^{\text {TM }}$ kit (TaKaRa, Japan) in an Applied Biosystems 7900 Fast Real-Time PCR system (Applied Biosystems, Foster City, California, USA). The relative expression of EYA4 mRNA was evaluated via the comparative cycle threshold (CT) method. GAPDH was used as an endogenous control to normalize the relative expression of EYA4. The primer sequences were as follows: EYA4 forward-5'AGATGGAGCGCTTGACACTT-3' and reverse-5'-TCCCCGAATACTGAGTCTGG-3'. GAPDH forward primer 5'CGGAGTCAACGGATTTGGTCGTAT-3', reverse primer 5'-AGCCTTCTCCATGGTGGTGAAGAC-3'. Each test was performed in triplicate.

\section{Statistical analysis}

Statistical analyses were performed using the software of SPSS 21.0 (SPSS Inc., Chicago, IL, USA) and GraphPad Prism 5 (GraphPad, San Diego, CA, USA). Continuous data were presented as mean \pm SD. The different levels of EYA4 mRNA between CRC tissues and adjacent normal tissues were analyzed with student's $t$ test. $\chi^{2}$ test was used to assess the relationship between EYA4 expression and the clinicopathological characteristics. Kaplan-Meier analysis with log rank test was used to evaluate the effects of EYA4 expression level on overall survival of CRC patients. Cox regression analysis was conducted to evaluate the prognostic value of EYA4 and clinical parameters in CRC. The difference was considered to be statistically significant when $P$ value less than 0.05 .

\section{Results}

\section{The expression of EYA4 was decreased in CRC tissues}

To study the expression pattern of EYA4 in CRC, qRT-PCR method was applied in this study. As shown in Fig. 1, the mRNA expression level of EYA4 was remarkably downregulated in CRC tissues compared with adjacent cancer tissues $(P<0.01)$.

\section{Relationship between EYA4 expression and clinicopathological characteristics of patients with CRC}

To investigate whether EYA4 was involved in the development of $\mathrm{CRC}$, the patients were divided into high expression group $(\mathrm{n}=99)$ and low expression group $(\mathrm{n}=56)$ according to the mean expression of EYA4. The expression of EYA4 was significantly associated with DUKE stage $(P=0.034)$, differentiation $(P=$ $0.027)$ and vascular invasion $(P=0.037)$. However, no correlation was found between EYA4 expression and age, gender, lymph node metastasis or tumor location ( $P>0.05$ for all) (Table 1$)$. 
To evaluate the prognostic potential of EYA4 in CRC, we analyzed the association between EYA4 expression and the overall survival using Kaplan-Meier analysis with the log-rank test. The survival curves showed that patients with low expression of EYA4 had a shorter overall survival than those with high expression (Fig. 2, log rank test, $P=0.008$ ). The univariate analysis revealed that lymph node metastasis $(\mathrm{HR}=2.037,95 \% \mathrm{Cl}=1.121-3.702, P=0.020)$, DUKES clinical stage $(\mathrm{HR}=1.819,95 \% \mathrm{Cl}=$ $1.006-3.289, P=0.048)$, and $E Y A 4$ expression $(\mathrm{HR}=2.179,95 \% \mathrm{Cl}=1.202-3.950, P=0.010)$ showed significant association with outcomes of patients with CRC. Multivariate analysis demonstrated that lymph node metastasis $(\mathrm{HR}=1.840,95 \% \mathrm{Cl}=1.005-3.366, P=0.048)$ and $E Y A 4$ expression $(\mathrm{HR}=1.989$, $95 \% \mathrm{Cl}=1.090-3.62902, P=0.025)$ were independent prognostic biomarkers for CRC patients (Table 2).

Table 2

Cox regression model for prognosis analysis in CRC patients

\begin{tabular}{|c|c|c|c|c|c|c|}
\hline \multirow[t]{2}{*}{ Characteristics } & \multicolumn{3}{|c|}{ Univariate analysis } & \multicolumn{3}{|c|}{ Multivariate analysis } \\
\hline & HR & $95 \% \mathrm{Cl}$ & $P$ & HR & $955 \mathrm{Cl}$ & $P$ \\
\hline Age & 1.510 & $0.823-2.773$ & 0.183 & - & - & - \\
\hline Gender & 1.061 & $0.578-1.946$ & 0.849 & - & - & - \\
\hline Lymph node metastasis & 2.037 & $1.121-3.702$ & 0.020 & 1.840 & $1.005-3.366$ & 0.048 \\
\hline DUKES Clinical stage & 1.819 & $1.006-3.289$ & 0.048 & - & - & - \\
\hline Differentiation & 1.293 & $0.699-2.392$ & 0.412 & - & - & - \\
\hline Tumor location & 0.992 & $0.540-1.823$ & 0.978 & - & - & - \\
\hline Vascular invasion & 1.512 & $0.832-2.746$ & 0.175 & - & - & - \\
\hline $\begin{array}{l}\text { EYA4 expression } \\
\text { (low vs high) }\end{array}$ & 2.179 & $1.202-3.950$ & 0.010 & 1.989 & $1.090-3.629$ & 0.025 \\
\hline
\end{tabular}

\section{Discussion}

CRC is a frequently diagnosed malignant tumor worldwide, with high morbidity and mortality [16]. In China, the incidence of CRC shows increasing tread, posing a great threat to human health [17]. Despite of the various available treatments for $\mathrm{CRC}$, the long term outcomes of CRC patients still remain unsatisfactory, which may be attributed to high occurrence of postoperative recurrence and metastasis [18]. Tumor progression prediction represents a challenge for CRC patients in clinic, due to the lack of credible biomarkers [19]. Therefore, identification of effective prognostic biomarkers for CRC may be a promising approach to improve management and prognosis of patients with CRC. 
The development and progression of CRC is a complex process, with the involvement of a variety of molecules and signaling pathways, such as oncogene activation, suppressor gene inactivation, and epigenetic modifications [20]. Up to now, the molecular mechanisms underlying CRC is still unclear. In order to improve the management and prognosis of $\mathrm{CRC}$, various researches were devoted to explore the genetic biomarkers for prognosis prediction in CRC. For instance, Zhu et al., found that Twist1 could enhance proliferation and chemoresistance of CRC cells which might be a potential prognostic marker and a molecular therapeutic target for the cancer [21]. Zhang et al. indicated that up-regulation of nemolike kinase (NLK) was positively correlated with recurrence and distant metastasis of CRC patients, predicting poor prognosis for patients with the disease [22]. The clinical significance of MTA2 expression for prognosis of CRC was investigated by Ding et al. They found the expression profile of MTA2 exhibited high in CRC tissues specimens, and significantly correlated with overall survival of the patients, suggesting its possible as a prognostic biomarker for CRC [23]. These cancer-related molecules might provide reliable information for tumor progression of $\mathrm{CRC}$. In the current study, we investigated the prognostic value of EYA4 for CRC.

EYA4, a member of EYA family, has recently been identified as a tumor suppressor gene in many kinds of cancers, including hepatocellular carcinoma, acute myeloid leukemia, oral cancer, etc $[14,24,25]$. In the current study, we investigated the functional roles of EYA4 in CRC. We detected the expression of EYA4 at mRNA level in CRC tumor tissues and the adjacent normal tissues. Our results showed that the level of EYA4 was significantly down-regulated in tissues compared with adjacent normal tissues. Then we further investigated the effects of EYA4 expression level on the development of CRC. The results indicated that EYA4 was negatively associated with DUKE stage, differentiation and vascular invasion. All the data revealed the anti-tumor action of EYA4 in CRC, and its reduced expression could contribute to malignant progression of the disease.

Given its function in tumorigenesis, EYA4 was identified as a biomarker for several cancers. In pancreatic ductal adenocarcinoma, down-regulation of EYA4 predicted short overall survival for the patients [26]. The expression pattern of EYA4 also held referenced significance for prognosis of patients with intrahepatic cholangiocarcinoma [27]. In CRC, Liu et al. reported that the serum methylation levels of EYA4 were significantly different between stage I CRC patients and healthy individuals that might be an useful biomarker for early detection of CRC [28]. However, the prognostic value of EYA4 in CRC was still unclear. In this study, Kaplan-Meier analysis showed that patients with low expression of EYA4 had a shorter overall survival than those with high expression. According to cox regression analysis, EYA4 might be employed as an independent prognostic factor for CRC. Low expression of EYA4 predicted poor prognosis for the patients.

\section{Conclusions}

In conclusion, EYA4 is decreased in CRC patients and its expression is significantly correlated with tumor differentiation, metastasis and clinical stage. EYA4 may be a potential prognostic biomarker for CRC. 


\section{Abbreviations}

Eyes absent 4 (EYA4)

colorectal cancer (CRC)

quantitative real-time polymerase chain reaction (qRT-PCR)

inflammatory bowel disease (IBD)

ulcerative colitis (UC)

cycle threshold (CT)

nemo-like kinase (NLK)

\section{Declarations}

\section{Ethics approval and consent to participate}

This study was supported by the Ethics Committee of Chinese PLA General Hospital and also has been carried out in accordance with the World Medical Association Declaration of Helsinki.

The subjects had been informed the objective. Certainly, written consents were signed by every subject in this study.

\section{Consent for publication}

We obtaining permission from participants to publish their data.

\section{Availability of data and materials}

The datasets used and/or analysed during the current study are available from the corresponding author on reasonable request.

\section{Competing interests}

The authors declare that they have no competing interests.

\section{Funding}

Not applicable.

\section{Authors' contributions}


Y.Y. design of the work; X.D. and S.X. the acquisition, analysis, S.L. and D.T. interpretation of data; S.H. and Y.W. the creation of new software used in the work; R.L. have drafted the work or substantively revised it. All authors read and approved the final manuscript.

\section{Acknowledgements}

Not applicable.

\section{References}

1. Siegel RL, Miller KD, Jemal A. Cancer statistics, 2015. Cancer J Clin. 2015;65(1):5-29.

2. Herrinton LJ, Liu L, Levin TR, Allison JE, Lewis JD, Velayos F. Incidence and mortality of colorectal adenocarcinoma in persons with inflammatory bowel disease from 1998 to 2010. Gastroenterology. 2012;143(2):382-9.

3. Jess T, Rungoe C, Peyrin-Biroulet L. Risk of colorectal cancer in patients with ulcerative colitis: a meta-analysis of population-based cohort studies. Clinical gastroenterology hepatology: the official clinical practice journal of the American Gastroenterological Association. 2012;10(6):639-45.

4. Wang Y, Duan B, Shen C, Wu B, Luo J, Zhao G. Treatment and multivariate analysis of colorectal cancer with liver metastasis. Hepato-gastroenterology. 2014;61(134):1568-73.

5. Lou X, Kang B, Zhang J, Hao C, Tian X, Li W, Xu N, Lu Y, Liu S. MFAP3L activation promotes colorectal cancer cell invasion and metastasis. Biochim Biophys Acta. 2014;1842(9):1423-32.

6. Li Y, Liang L, Dai W, Cai G, Xu Y, Li X, Li Q, Cai S. Prognostic impact of programed cell death-1 (PD-1) and PD-ligand 1 (PD-L1) expression in cancer cells and tumor infiltrating lymphocytes in colorectal cancer. Mol Cancer. 2016;15(1):55.

7. De Rosa M, Pace U, Rega D, Costabile V, Duraturo F, Izzo P, Delrio P. Genetics, diagnosis and management of colorectal cancer (Review). Oncol Rep. 2015;34(3):1087-96.

8. Tootle TL, Silver SJ, Davies EL, Newman V, Latek RR, Mills IA, Selengut JD, Parlikar BE, Rebay I. The transcription factor Eyes absent is a protein tyrosine phosphatase. Nature. 2003;426(6964):299302.

9. Borsani G, DeGrandi A, Ballabio A, Bulfone A, Bernard L, Banfi S, Gattuso C, Mariani M, Dixon M, Donnai $D$, et al. EYA4, a novel vertebrate gene related to Drosophila eyes absent. Human molecular genetics. 1999;8(1):11-23.

10. Tadjuidje E, Hegde RS. The Eyes Absent proteins in development and disease. Cell Mol Life Sci. 2013;70(11):1897-913.

11. Ecsedi S, Hernandez-Vargas H, Lima SC, Vizkeleti L, Toth R, Lazar V, Koroknai V, Kiss T, Emri G, Herceg Z, et al. DNA methylation characteristics of primary melanomas with distinct biological behaviour. PloS one. 2014;9(5):e96612.

12. Selamat SA, Galler JS, Joshi AD, Fyfe MN, Campan M, Siegmund KD, Kerr KM, Laird-Offringa IA. DNA methylation changes in atypical adenomatous hyperplasia, adenocarcinoma in situ, and lung 
adenocarcinoma. PloS one. 2011;6(6):e21443.

13. Kisiel JB, Yab TC, Taylor WR, Chari ST, Petersen GM, Mahoney DW, Ahlquist DA. Stool DNA testing for the detection of pancreatic cancer: assessment of methylation marker candidates. Cancer. 2012;118(10):2623-31.

14. Hou X, Peng JX, Hao XY, Cai JP, Liang LJ, Zhai JM, Zhang KS, Lai JM, Yin XY. DNA methylation profiling identifies EYA4 gene as a prognostic molecular marker in hepatocellular carcinoma. Ann Surg Oncol. 2014;21(12):3891-9.

15. Kim SJ, Tae CH, Hong SN, Min BH, Chang DK, Rhee PL, Kim JJ, Kim HC, Kim DH, Kim YH. EYA4 Acts as a New Tumor Suppressor Gene in Colorectal Cancer. Molecular carcinogenesis. 2015;54(12):1748-57.

16. Schreuders EH, Ruco A, Rabeneck L, Schoen RE, Sung JJ, Young GP, Kuipers EJ. Colorectal cancer screening: a global overview of existing programmes. Gut. 2015;64(10):1637-49.

17. Chen W, Zheng R, Baade PD, Zhang S, Zeng H, Bray F, Jemal A, Yu XQ, He J. Cancer statistics in China, 2015. Cancer J Clin. 2016;66(2):115-32.

18. Bardelli A, Siena S. Molecular mechanisms of resistance to cetuximab and panitumumab in colorectal cancer. Journal of clinical oncology: official journal of the American Society of Clinical Oncology. 2010;28(7):1254-61.

19. Li C, Zhou L, He J, Fang XQ, Zhu SW, Xiong MM. Increased long noncoding RNA SNHG20 predicts poor prognosis in colorectal cancer. BMC Cancer. 2016;16:655.

20. Liu DR, Guan QL, Gao MT, Jiang L, Kang HX. miR-1260b is a Potential Prognostic Biomarker in Colorectal Cancer. Medical science monitor: international medical journal of experimental clinical research. 2016;22:2417-23.

21. Zhu DJ, Chen XW, Zhang WJ, Wang JZ, Ouyang MZ, Zhong Q, Liu CC. Twist1 is a potential prognostic marker for colorectal cancer and associated with chemoresistance. American journal of cancer research. 2015;5(6):2000-11.

22. Zhang W, He J, Du Y, Gao XH, Liu Y, Liu QZ, Chang WJ, Cao GW, Fu CG. Upregulation of nemo-like kinase is an independent prognostic factor in colorectal cancer. World journal of gastroenterology: WJG. 2015;21(29):8836-47.

23. Ding W, Hu W, Yang H, Ying T, Tian Y. Prognostic correlation between MTA2 expression level and colorectal cancer. Int J Clin Exp Pathol. 2015;8(6):7173-80.

24. Huang S, Jiang MM, Chen GF, Qian K, Gao HH, Guan W, Shi JL, Liu AQ, Liu J, Wang BH, et al. Epigenetic Silencing of Eyes Absent 4 Gene by Acute Myeloid Leukemia 1-Eight-twenty-one Oncoprotein Contributes to Leukemogenesis in t(8;21) Acute Myeloid Leukemia. Chin Med J. 2016;129(11):1355-62.

25. Towle R, Truong D, Garnis C. Epigenetic mediated silencing of EYA4 contributes to tumorigenesis in oral dysplastic cells. Genes Chromosom Cancer. 2016;55(7):568-76.

26. Mo SJ, Liu X, Hao XY, Chen W, Zhang KS, Cai JP, Lai JM, Liang LJ, Yin XY. EYA4 functions as tumor suppressor gene and prognostic marker in pancreatic ductal adenocarcinoma through beta- 
catenin/ID2 pathway. Cancer letters. 2016;380(2):403-12.

27. Hao XY, Cai JP, Liu X, Chen W, Hou X, Chen D, Lai JM, Liang LJ, Yin XY. EYA4 gene functions as a prognostic marker and inhibits the growth of intrahepatic cholangiocarcinoma. Chinese journal of cancer. 2016;35(1):70.

28. Liu Y, Tham CK, Ong SY, Ho KS, Lim JF, Chew MH, Lim CK, Zhao Y, Tang CL, Eu KW. Serum methylation levels of TAC1. SEPT9 and EYA4 as diagnostic markers for early colorectal cancers: a pilot study. Biomarkers: biochemical indicators of exposure response susceptibility to chemicals. 2013;18(5):399-405.

\section{Figures}

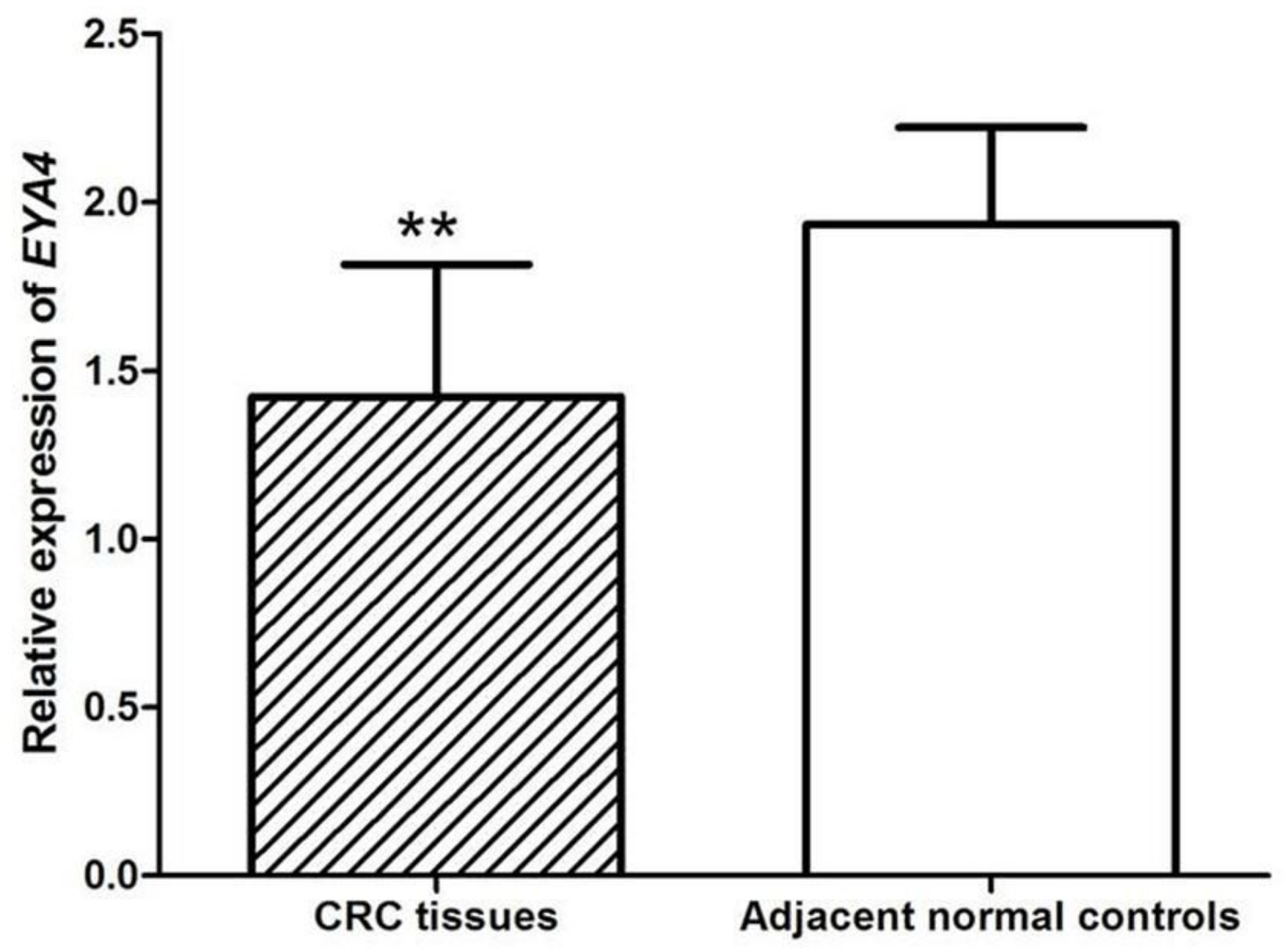

Figure 1

The relative expression level of EYA4 mRNA in CRC tissues and adjacent normal tissue. The expression level of EYA4 in CRC tissues was lower than that in the normal specimens (**: suggested $\mathrm{P}<0.01$ ). 


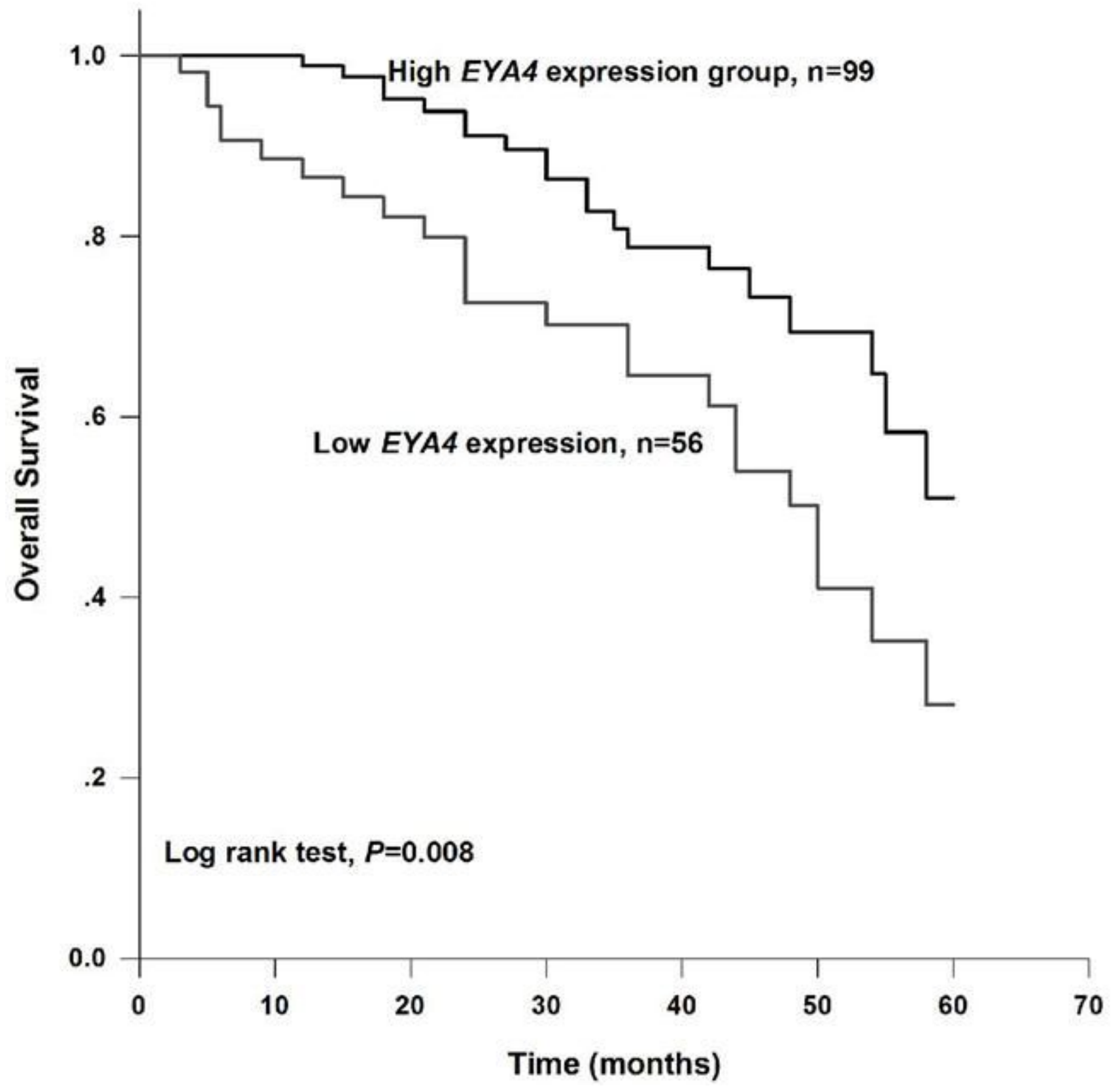

Figure 2

Kaplan-Meier analysis for overall survival of patients with CRC. Patients with low EYA4 expression had a shorter overall survival than those high expression (log rank test, $\mathrm{P}=0.008$ ). 